\title{
Bien-être et élevage des palmipèdes
}

\author{
D. GUÉMENE' 1 , G. GUY², L. MIRABITO 3 , J. SERVIÈRE 4 , J-M. FAURE 1 \\ ${ }^{1}$ INRA, UR83 Recherches Avicoles, F-37380 Nouzilly, France \\ 2 INRA, UE89 Palmipèdes à Foie gras, Artiguères, F-40280 Benquet, France \\ ${ }^{3}$ ITAVI, 28 rue du Rocher, F-75008 Paris, France \\ ${ }^{4}$ INRA, INA-PG, UMR791 Physiologie de la nutrition et alimentation, 16 Rue Claude Bernard, F-75231 Paris, France \\ Courriel : Daniel.Guemene@tours.inra.fr
}

\begin{abstract}
Plusieurs espèces d'oie et de canards sont élevées en France à des fins de production d'animaux à rôtir (filière maigre) et de foie gras (filière gras). La production d'oie est marginale $(<5 \%)$ et les espèces concernées sont essentiellement le canard de barbarie pour la filière «Palmipèdes maigres» et le canard mulard mâle pour la filière «Palmipèdes gras». Leurs conditions d'élevage ont évolué et divers systèmes de production coexistent avec, pour chacun, des contraintes spécifiques. Par ailleurs, ces espèces ont des besoins comportementaux différents. La production de foie gras focalise la majorité des critiques, aussi notre propos concernera-t-il essentiellement les résultats que nos travaux apportent aux questionnements relatifs au bien-être, résultant de la pratique du gavage. Ceux associés à la filière maigre seront succinctement évoqués, de même que le cadre législatif dans lequel s'inscrivent ces productions.
\end{abstract}

\section{1 / Les différentes espèces et productions}

La production française de viande de canard (250 $000 \mathrm{~T}$ équivalent carcasse) représente plus de $50 \%$ de la production européenne alors que celle de foie gras $\left(\begin{array}{llll}18 & 000 & \mathrm{~T}\end{array}\right)$ représente environ $80 \%$ de la production mondiale. Le canard de barbarie, l'espèce privilégiée pour la production de canards à rôtir, représente environ $45 \%$ du tonnage de viande de canard pour un effectif annuel d'environ 55 millions de canards. Le canard mulard mâle, un hybride entre un mâle Barbarie et une cane Pékin, avec un effectif d'environ 35 millions de canards en 2005, est quant à lui à l'origine de près de $95 \%$ de la production française de foie gras. La production de viande associée, dont les magrets, représente $55 \%$ du tonna- ge de viande de canard. La production de foie gras est une spécificité française. En Europe, la Hongrie (foie gras d'oie) et la Bulgarie ont une production significative ; l'Espagne et la Belgique ayant des productions modestes, mais en progression. L'élevage de palmipèdes dans les autres pays Européens concerne essentiellement le canard Pékin, utilisé à des fins de production de viande à rôtir. L'oie grasse $(850000$ T/an), principalement de souche landaise est utilisée en France pour la production de foie gras (600 T/an), alors que les oies du Rhin et blanches d'Italie (500 $000 \mathrm{~T} / \mathrm{an}$ ), sont utilisées pour la production d'oies à rôtir.

\section{2 / Le cadre législatif}

La législation s'appliquant aux productions avicoles a fait l'objet d'une synthèse récente (Guémené et Faure 2004). Les palmipèdes ont fait l'objet de trois recommandations, adoptées en 1999 et concernant l'oie domestique (Anser sp.) (T-AP [95/5]), le canard commun (Anas platyrhynchos) (T-AP [94/3]) et le canard de barbarie (Cairina moschata) et le canard mulard (T-AP [95/20]). Elles devraient s'appliquer aux nouvelles installations, lors du remplacement des existantes depuis le $31 / 12 / 2004$, et pour toutes à partir du 31/12/2010, mais n'ont à ce jour fait l'objet d'aucune transcription en droit communautaire ou national. Concrètement, l'utilisation de sol en caillebotis intégral et celle de la cage individuelle sont proscrites. Les pratiques d'épointage du bec et de dégriffage ne sont tolérées que dans un contexte très restrictif, pour les seuls canards de barbarie et mulard. Concernant l'épointage du bec, la tolérance ne concerne que le crochet de la mandibule supérieure et il doit être réalisé avant l'âge de 10 jours. Les restrictions alimentaires, les photopériodes et les rythmes lumineux particuliers devraient être interdits. Enfin, les systèmes d'abreuvement devraient permettre aux palmipèdes de se couvrir la tête avec de l'eau. La production de foie gras ne pourra être poursuivie que dans les zones où elle était pratiquée à la date d'adoption des recommandations. L'Italie et la Pologne l'ont officiellement interdite et elle l'est de facto dans les pays membres du Conseil de l'Europe ayant ratifié la Convention de 1976, où elle n'était pas alors pratiquée.

\section{3 / Bien-être dans la filière «Palmipèdes maigres»}

Les problèmes de bien-être de la filière «Palmipèdes maigres» ont fait l'objet d'une synthèse récente (Rodenburg et al 2005). En France, les élevages sont majoritairement de type standard, c'est-à-dire avec des bâtiments en claustration totale, souvent sur caillebotis intégral, avec des densités élevées ( $>$ à $50 \mathrm{~kg} / \mathrm{m}^{2}$ en fin de bande). Les groupes sont de grande taille (3000 à 10000$)$ et soumis à des intensités lumineuses faibles, pour limiter picage et cannibalisme. Ce problème reste la préoccupation majeure de l'élevage du canard de barbarie et il est actuellement indispensable de procéder à l'épointage du bec et au dégriffage. Ces interventions sont réalisées, actuellement vers l'âge de 21 jours. L'application à l'éclosion d'un traitement du bec par infrarouge est en cours de développement et les premiers résultats semblent satisfaisants. Pour les canards pékin, l'épointage est souvent nécessaire pour les reproducteurs. Par ailleurs, de nombreux facteurs peuvent affecter le bien-être des canards et, chaque fois que cela est possible, il convient de proposer des aménagements permettant d'améliorer les 
conditions d'élevage. Concernant les oies à rôtir, souvent élevées en groupes de petites tailles ayant accès à des parcours, leurs modes d'élevage ne suscitent pas de critiques particulières en terme de bien-être.

\section{4 / Bien-être dans la filière «Palmipèdes gras»}

La production de foie gras est fortement remise en cause par les associations de protection animale. Afin d'évaluer l'impact de cette pratique sur le bien-être des palmipèdes, des études ont été conduites depuis une dizaine d'années pour analyser les réponses comportementales et physiologiques des palmipèdes au gavage (Guémené et Guy 2004).

\section{1 / Mode d'élevage des palmi- pèdes destinés au gavage}

L'élevage d'un canard destiné à la production de foie gras comporte trois phases. Pendant la phase de démarrage, d'une durée d'environ 4 semaines, les canetons, installés dans un bâtiment chauffé, sont alimentés à volonté. Lui succède la phase de croissance, d'une durée de huit semaines. Les canards peuvent avoir accès à un parcours, où ils disposent alors individuellement d'une surface de 3 à $5 \mathrm{~m}^{2}$. L'alimentation est successivement distribuée à volonté, puis par repas en quantités limitées. Les quantités sont progressivement augmentées au cours de la dernière semaine. Ce régime permet de préparer le canard au gavage, en stimulant la consommation volontaire, en induisant une distension de l'œsophage, ainsi qu'un début de stéatose hépatique. A environ 12 semaines d'âge, les canards sont transférés, le plus souvent en cage individuelle, dans des bâtiments dont l'ambiance est contrôlée. Ils y restent en moyenne 2 semaines, pendant lesquelles ils sont gavés deux fois par jour. L'acte de gavage consiste à introduire un tube (embuc) dans l'œsophage, pour y déposer de l'aliment en quantité croissante. Chez l'oie, le gavage débute selon les cas entre 14 et 18 semaines d'âge, se prolonge généralement 3 semaines et implique de 3 à 5 repas quotidiens.

Les interrogations relatives au bienêtre des palmipèdes «gras» concernent essentiellement la phase de gavage, en particulier, les incidences de l'acte de gavage (ingestion, stress, douleur, mortalité) et de son environnement (homme et logement).

\section{2 / Quelles sont les capacités d'ingestion des palmipèdes ?}

Les quantités d'aliment distribuées aux palmipèdes lors du gavage sont augmentées progressivement. De $250 \mathrm{~g}$ le premier jour, les quantités maximales peuvent atteindre $450 \mathrm{~g}$ par repas, soit de 8 à $12 \mathrm{~kg}$ au total pour la période de gavage (Chalimbaud 2004, figure 1). Ces chiffres, certes élevés, sont à comparer avec la consommation spontanée de canards mulards qui peut transitoirement atteindre plus de $750 \mathrm{~g}$ (Guy et al 1998, figure 1). Les oies pour leur part peuvent ingérer quotidiennement jusqu'à $1 \mathrm{~kg}$ de fourrage en sus de leur alimentation (Leprettre et al 2000). Le canard mulard et l'oie peuvent donc ingérer des quantités d'aliment comparables, voire supérieures, à celles qui leur sont distribuées lors d'un repas par gavage. Cette aptitude à surconsommer ne permet pas pour autant de proposer actuellement une alternative au gavage, même si des approches expérimentales prospectives sont explorées. Elle permet par contre d'en réduire la durée.

\section{3 / L'acte de gavage est-il perçu comme un stress ?}

Nous avions émis l'hypothèse que l'acte de gavage pouvait être à l'origine de stress aigus dont la répétition pourrait induire un stress chronique, surtout chez des canards confinés dans des cages individuelles exiguës. Or, cet acte n'induit généralement pas d'augmentation de la corticostéronémie chez le canard mulard, s'il est placé en cage individuelle (Guémené et al 2001,
2006, figure 2). Par contre, le canard mulard est sensible à la capture, la contention ou la suspension. La diminution progressive d'amplitude des réponses de corticostérone lors de la répétition de ces stress révèle toutefois une accoutumance rapide (Guémené et al 2006). Une telle évolution est également observée lorsque la réalisation de l'acte de gavage nécessite capture et immobilisation préalable, c'est-à-dire lorsque les canards sont élevés collectivement en cage ou au sol (Guémené et al 2006, figure 2). En effet, dans ce contexte, une élévation significative de la corticostéronémie est mesurée après le premier gavage et le test à l'ACTH suggère pour les canards élevés au sol, un état de stress chronique, ce qui valide par ailleurs l'utilisation de la corticostéronémie comme indicateur de stress chez les palmipèdes. Chez l'oie, bien qu'aucune indication de stress chronique n'ait pu être mise en évidence, le gavage en parc collectif induit également une élévation de la corticostéronémie, indicatrice d'un stress aigu, lors des premiers repas (Guémené et al 1999).

\section{4 / L'acte de gavage est-il dou- loureux ?}

Douleur et souffrance sont difficiles à évaluer chez l'animal parce qu'elles impliquent des composantes émotionnelle et psychologique. Il est donc préférable d'utiliser le concept de nociception (réception de stimulations nocives) qui correspond à l'expérience sensorielle déclenchant des réactions fonctionnelles et comportementales qui, elles, sont mesurables.

Figure 1. Evolutions quotidiennes des quantités ingérées spontanément par des canards mulards mâles recevant des aliments, qui diffèrent par leur composition, ou au cours de la période de gavage (courbe de gavage) (Moyenne).

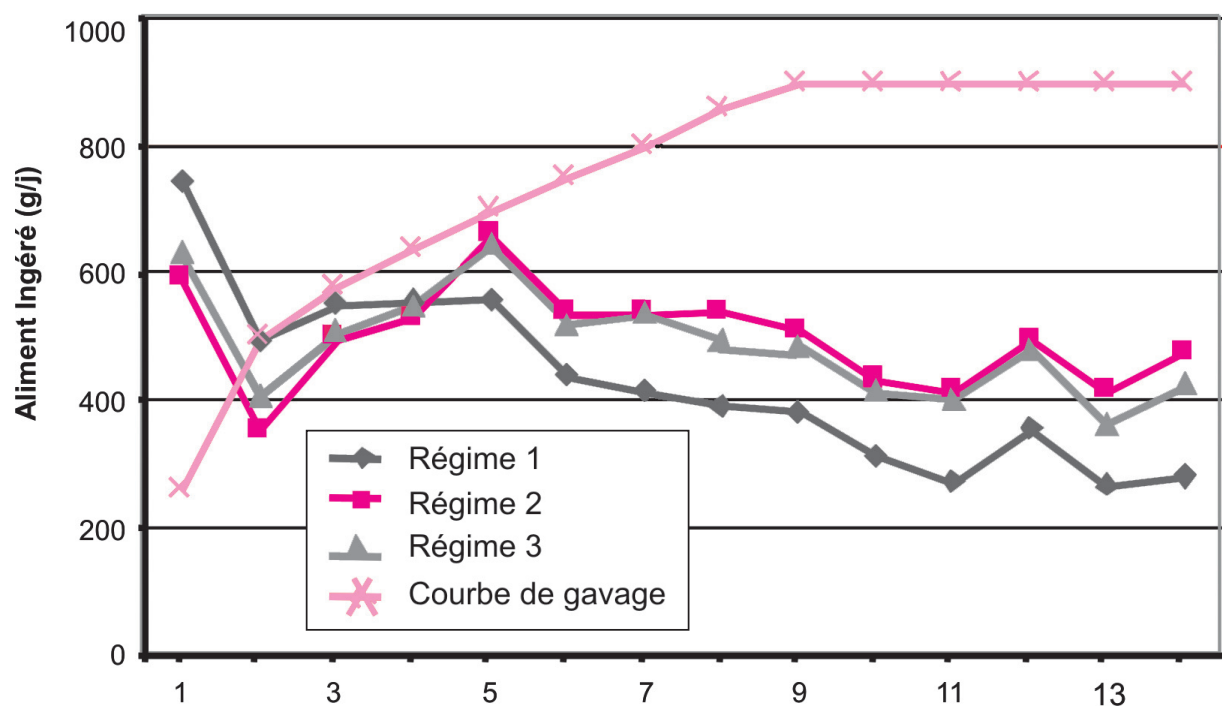


Figure 2. Concentrations en corticostérone ( $\mathrm{ng} / \mathrm{mL}$ plasma) durant la période d'élevage.

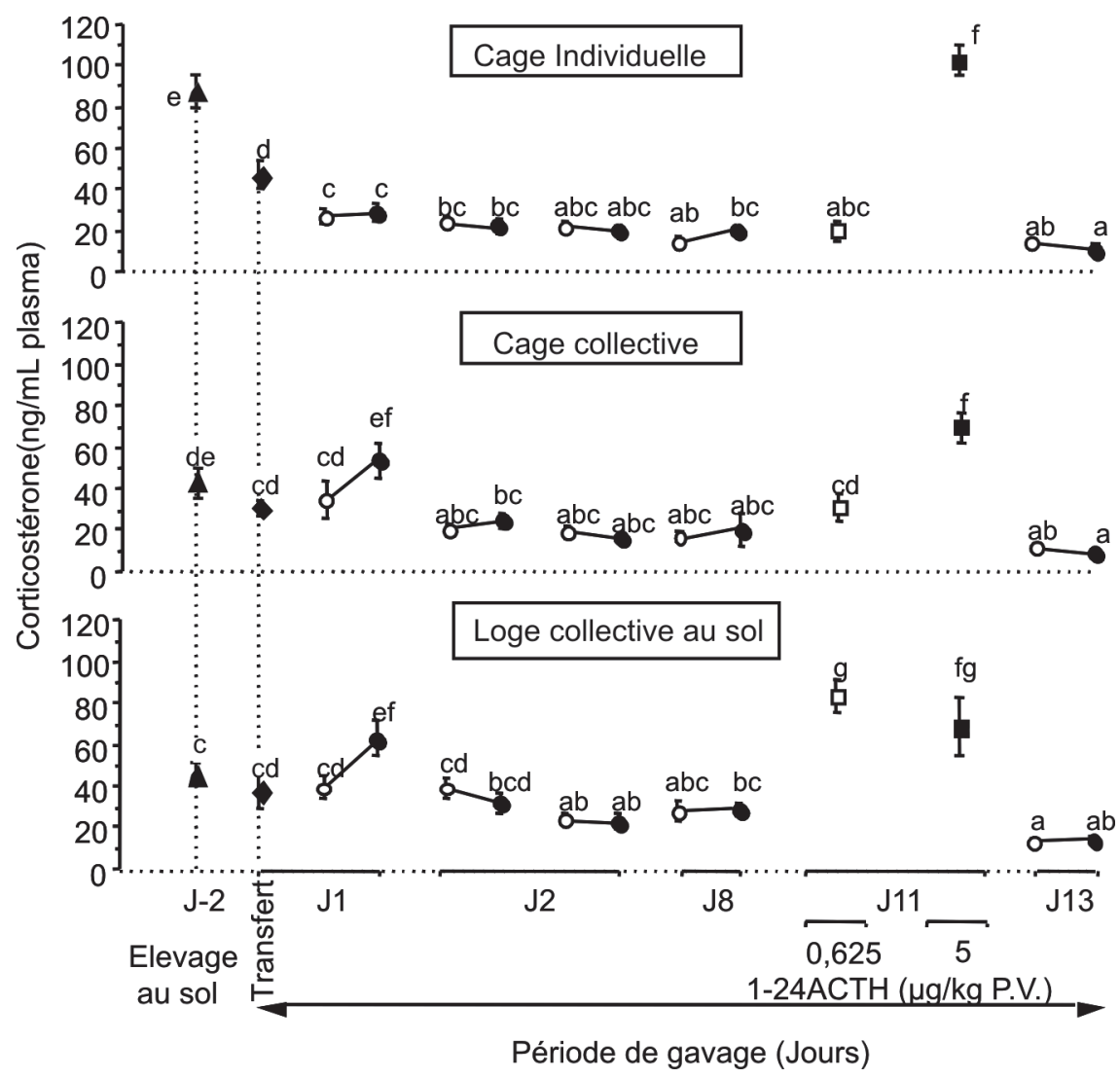

pré-gavage $(\boldsymbol{\Delta})$, au moment du transfert $(\diamond)$, et au cours de la période de gavage $[75 \mathrm{mn}$ avant (O) et $15 \mathrm{mn}$ après gavage $(\bullet)$ ou $15 \mathrm{mn}$ après injection de 1-24ACTH aux doses de 0,625 ( $\square$ ) ou $5 \mu \mathrm{g} / \mathrm{kg}$ P.V. ( $\square)$ ] chez des canards mulards hébergés en cages individuelles ou collectives et au sol en loge collective après transfert. (Moyenne \pm E.T M.)

a, b.....g : les moyennes affectées de lettres différentes pour un groupe expérimental spécifique diffèrent significativement $(P<.05)$.

Le tube digestif supérieur des oiseaux (figure 3), contrairement à celui des mammifères, présente un orifice dégagé (ouverture trachéale au milieu de la langue) et des parois souples (Marshall 1960). Au niveau du cou, le jabot des palmipèdes est une poche fusiforme de stockage dont les parois avec replis longitudinaux sont extensibles et recouvertes d'une couche kératinisée assurant une protection mécanique. Ces spécificités permettent à certaines espèces d'avaler des aliments de grande taille, comme l'illustre la citation d'Audubon (Elphick et al 2001) «birds have a remarkable ability to expand the mouth and strech the oesophagus to swallow large prey». En dépit de ces caractéristiques anatomiques, nous ne pouvons totalement exclure que les stimuli associés aux embucquages répétés puissent déclencher des foyers inflammatoires, avec augmentation de la perméabilité des parois vasculaires et extravasation, favorisant l'émission de signaux nociceptifs viscéraux. Des résultats en cours de publication permettront d'éclaircir ce point très prochainement.
L'hypertrophie du foie est souvent considérée comme un état pathologique et une source de douleur. Chez les oiseaux, le foie est le site majeur de la lipogenèse (Hermier 1997) alors qu'elle a lieu majoritairement dans le tissu adipeux chez les mammifères. En raison de cette particularité, l'augmentation de la quantité de lipides dans le foie ne signe pas une pathologie, mais correspond à une stéatose nutritionnelle. Cette stéatose hépatique n'entraîne pas de nécrose ou de dégénérescence cellulaire (Hermier et al 1999) et est totalement réversible tant chez l'oie, que le canard (Babilé et al 1998, Bénard et al 2006). Concernant l'implication nociceptives de cette hypertrophie, il a été montré chez les mammifères que de nombreux organes internes comme le foie sont innervés par des récepteurs liés au système nerveux autonome, essentiellement les nerfs vague et glossopharyngien, qui sont à l'origine de réponses motrices réflexes et autonomes, mais ne déclenchent pas de sensation consciente (Cervero et Laird 1999). A notre connaissance, il n'existe pas d'études de neurophysiologie similaires chez les oiseaux, mais des descriptions anatomiques de l'innervation viscérale, montrent que, de la même façon, la majorité des fibres nerveuses présentent dans le foie appartiennent au système végétatif autonome (Chitescu et al 1976). Ainsi, par analogie avec l'innervation chez l'homme et sur la base des témoignages cliniques, on peut inférer que le foie ne peut être

Figure 3. Représentation schématique du tractus digestif du canard.

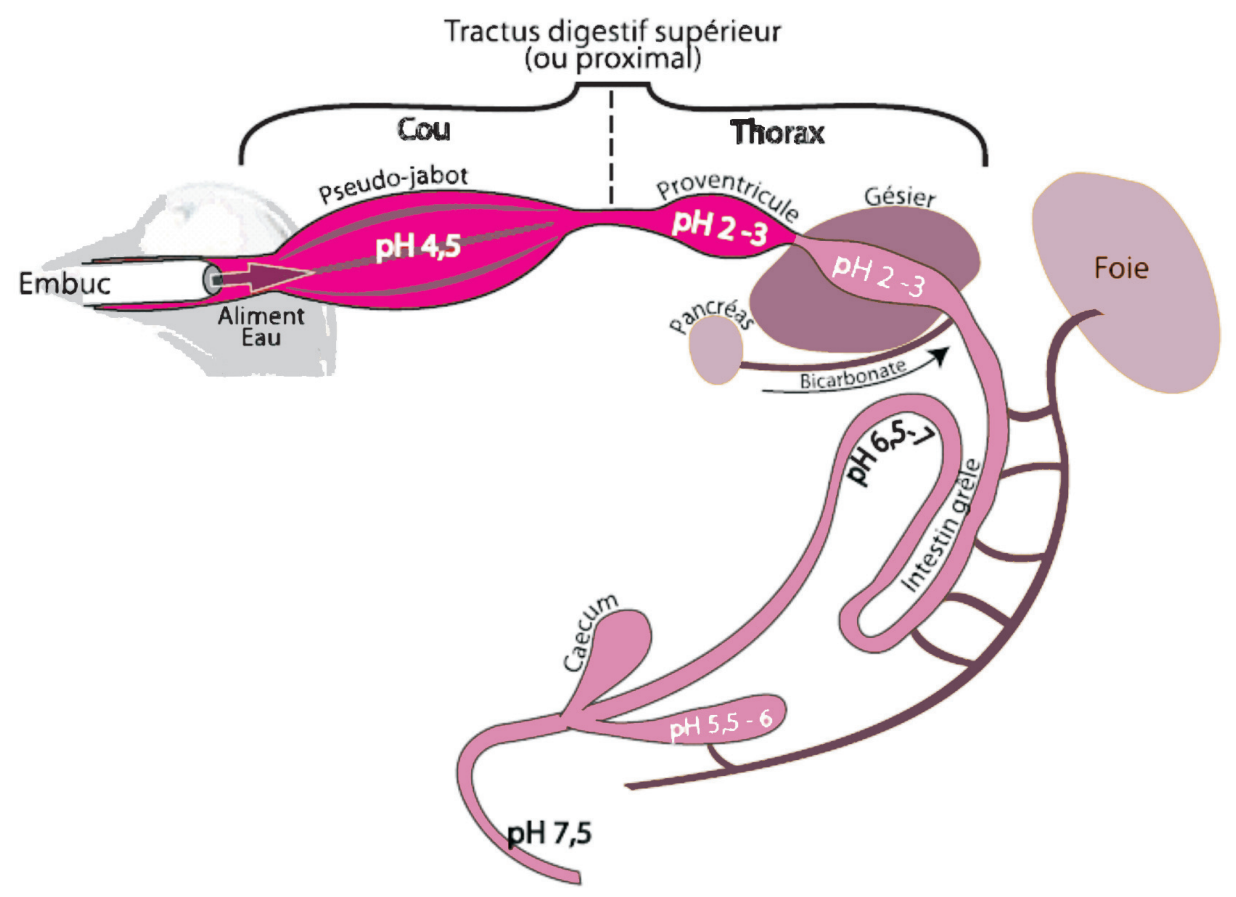


à l'origine directe de sensation nociceptive chez les oiseaux.

Le halètement des canards, qui pourrait être interprété comme un indicateur de souffrance, résulte d'un réflexe régulateur de la thermogenèse. Les oiseaux n'ont pas de glandes sudoripares et leur capacité d'élimination d'extrachaleur est limitée par les propriétés isolantes du plumage ; ils doivent donc ouvrir le bec et haleter pour l'éliminer. Cette hyperventilation est d'autant plus marquée que la charge énergétique nutritive est forte et pour cette raison augmente pendant la période de gavage (Guémené et al 2006).

\section{5 / Mortalité pendant la pério- de de gavage}

La mortalité fait débat et ne doit pas être éludée. Les résultats du terrain indiquent que la diminution du cheptel qui inclut, outre la mortalité, l'élimination d'animaux pour diverses causes, était en 2005 , inférieure à $2,5 \%$, au cours de chacune des phases d'élevage et gavage (Azard 2006). L'amélioration des techniques et des conditions d'hébergement, ainsi que la diminution des objectifs de production ont contribué à une réduction progressive de ce taux. Une analyse des causes d'élimination et de mortalité reste cependant à conduire pour mieux apprécier l'impact réel du gavage sur ce paramètre.

\section{6 / L'homme, l'animal et son environnement}

a) La relation entre l'éleveur-gaveur et les palmipèdes

Des tests comportementaux visant à détecter une éventuelle aversion vis-àvis du gaveur et/ou du gavage ont été réalisés en partant du principe qu'un évitement est observé si les stimuli associés à la situation sont aversifs. Des animaux ont été entraînés à se déplacer depuis une loge d'élevage, vers une loge d'alimentation. A l'issue de la période d'apprentissage (conditionnement opérant), ils ont été alimentés par gavage. Lors de la phase de test, les oies gavées ont continué à se déplacer spontanément et à la même vitesse que les oies témoins non gavées (Faure et al
2001). Chez le canard mulard, la réponse a été moins homogène, $y$ compris chez les animaux témoins ; la sortie spontanée de la loge d'élevage n'étant pas systématique (Faure et al 2001).

La réponse à l'homme a, quant à elle, été estimée en mesurant la distance à partir de laquelle des canards logés en cage individuelle manifestent un mouvement de recul à l'approche du gaveur ou d'une personne étrangère. Cette distance décroît au cours du gavage ; elle reste toujours inférieure à celle mesurée vis-à-vis d'un étranger (Faure et al 2001).

\section{b) Hébergement des canards durant} la phase de gavage

L'utilisation de la cage individuelle, pour le canard mulard, a été généralisée au cours des 20 dernières années $(85 \%$ des canards en 2005, Azard 2006). Ce système permet une contention aisée et son usage, associé à des évolutions du matériel de gavage, a contribué à une amélioration des conditions de travail du gaveur et de la productivité. Les canards placés en cages individuelles sont plus actifs et n'expriment pas de comportements stéréotypés (Guémené et al 2006). En revanche, ils y expriment apparemment des besoins supérieurs en termes de thermorégulation puisque des fréquences de halètement et d'abreuvement sont alors supérieures. Enfin, cette cage est à l'origine de restrictions comportementales, puisque le canard ne peut y adopter certains comportements et postures comme se retourner, se déplacer ou étirer ses ailes et battre des ailes.

En logement collectif, les réponses comportementales des animaux dépendent de la surface disponible (1000 vs 1500 ou $2000 \mathrm{~cm}^{2} /$ canard), indépendamment de la taille du groupe (3, 6 ou 9 canards), à l'exception des étirements d'aile dont la fréquence semble dépendre d'une interaction entre les deux facteurs, c'est-à-dire de l'espace total disponible (Mirabito et al 2002a, 2002b). Les données endocriniennes suggèrent qu'il est sans doute préférable d'avoir des groupes de tailles limitées, par ailleurs moins difficiles à gérer pour le gaveur (Mirabito et al 2002c). Les conditions d'hébergement doivent cependant faire l'objet de mises au point et d'évaluations complémentaires, puisqu'au sein des élevages, la mortalité et le nombre d'animaux présentant des traces de griffures sont généralement plus élevés en logement collectif (Mirabito et Sazy 2004). Les résultats diffèrent selon les zones corporelles pour les hématomes et la nature des lésions du bréchet. La propreté du plumage des canards ne semble quant à elle pas liée au caractère collectif ou individuel du logement, mais à la conception des équipements. Des investigations complémentaires, pour la mise au point de systèmes collectifs satisfaisants pour le bien-être des canards et appropriée d'un point de vue ergonomique pour le gaveur, sont en cours.

\section{Conclusion}

La production de foie gras est devenue emblématique de la perception négative que le citoyen peut se faire de certaines conditions d'élevage. Apporter des données expérimentales au débat éthique sur la pratique du gavage a donc motivé notre démarche scientifique. Dans l'état actuel de nos connaissance et sur la base des indicateurs physiologiques ou comportementaux utilisés, les expériences conduites à ce jour ne permettent pas d'affirmer que cette pratique nuit gravement au bien-être des palmipèdes, au sens où elle serait une cause majeure de stress, peur et douleur, voire de lésions et pathologie. En termes de perspectives, les investigations concernant la nociception sont actuellement complétées par des approches comportementale et neurophysiologique. Parallèlement, une approche «système» est mise en œuvre pour l'optimisation des cages collectives. Enfin, à titre plus prospectif, la réflexion, faisant appel aux connaissances issues d'approches mécanistes, comportementales et génétiques, et visant à explorer les possibilités éventuelles d'alternatives au gavage permettant de produire du foie gras, va être poursuivie. 


\section{Références}

Azard A., 2006. Résultats technico-économiques des éleveurs et gaveurs de palmipèdes à foie gras en 2005. Programme «Renapalm», 7 èmes Journ. Rech. Palm. Foie Gras, 219-222.

Babilé R., Auvergne A., Dubois J.P., Bénard G., Manse H., 1998. Réversibilité de la stéatose

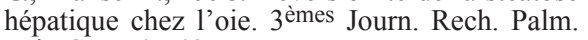
Foie Gras, 45-48.

Bénard G., Bengone T., Prehn D., Durand S., Labie C., Bénard P., 2006. Contribution à l'étude de la physiologie du canards en gavage : étude de la stéatose hépatique. Bull. Acad. Vét. Fr., 159 (1), 43-51.

Cervero F., Laird J., 1999. Visceral pain. The Lancet, 353, 2145-2148.

Chalimbaud J., 2004. Résultats technico-économiques des éleveurs et gaveurs de palmipèdes à foie gras : résultats 2003 du programme «Renapalm», 6èmes Journ. Rech. Palm. Foie Gras, 105-112.

Chitescu S.T., Cotofan V., Hillebrand A., 1976. Atlas d'anatomie des oiseaux domestiques. Editura Academiei Republicii Socialiste (Ed), Bucuresti, Romania, 295p

Elphick C., Dunning Jr J., Sibley D., 2001. The sibley guide to bird life and behaviour, $1^{\text {st }}$ Ed,. Helm C., Chanticleer Press Ltd (Eds) London, UK, 588p.

Faure J.M., Guémené D., Guy G., 2001. Is there avoidance of the force feeding of ducks and geese? Anim. Res., 50, 157-164.

Guémené D., Faure J.M., 2004. Productions avicoles : bien-être et législation européenne. INRA Prod Anim., 17, 59-68.
Guémené D., Guy G., 2004. The past, present and future of force-feeding and «foie-gras» production. Wld's Poult.Sci. J., 60, 210-222.

Guémené D., Guy G., Noirault J., Destombes N., Samson M., Gouraud P., Garreau-Mills M. Faure J.M., 1999. Physiological and behavioural responses to force-feeding procedure in male mule ducks and ganders. 1st W1d Waterfowl Conf., Taichung, Taiwan, 1-4 Dec., 413-424

Guémené D., Guy G., Noirault J., GarreauMills M., Gouraud P., Faure J.M., 2001. Force feeding procedures and physiological indicators of stress in male mule ducks. Brit. Poult. Sci., 42, 650-657.

Guémené D., Guy G., Noirault J., Destombes N., Faure J.M., 2006. Rearing conditions during the force-feeding period in male mule ducks and their impact upon stress and welfare. Anim. Res. 55, 443-458.

Guy G., Faure J.M., Guémené D., 1998. Capacité d'ingestion chez le canard mulard mâle. 3 èmes Journ. Rech. Palm. Foie Gras, 59-62.

Hermier D., 1997. Lipoprotein metabolism and fattening in poultry. J. Nut., 127, 805S-808S.

Hermier D., Salichon M.R., Guy G., Peresson R., Mourot J., Lagarrigue S., 1999. La stéatose hépatique des palmipèdes gavés : bases métaboliques et sensibilité génétique. INRA Prod Anim., 12, 265-271.

Leprettre S., Dubois J.P., Lavigne F., Babilé R., 2000. Densité en élevage et performances de croissance chez l'oie destinée au gavage. 4 èmes Journ. Rech. Palm. Foie Gras, 109-116.
Marshall A.J., 1960. Biology and comparative physiology of birds. Marshall A.J (Ed) Academic Press NY, London, UK, 1, 518p.

Mirabito L., Sazy E., 2004. Le logement collectif : vers un compromis entre le bien-être des canards et le confort des éleveurs. 6èmes Journ. Rech. Palm. Foie Gras, 105-112.

Mirabito L., Sazy E., Herault F., Guémené D., Faure J.M., Guy G., 2002a. Effet de la taille du groupe et de la surface allouée pendant la phase de gavage chez le canard mulard. I. Performances zootechniques. 5èmes Journ. Rech. Palm. Foie Gras, 76-79.

Mirabito L., Sazy E., Herault F., Guémené D., Faure J.M., Guy G., 2002b. Effet de la taille du groupe et de la surface allouée pendant la phase de gavage chez le canard mulard. II. Comportement. 5èmes Journ. Rech. Palm. Foie Gras, 80-83.

Mirabito L., Sazy E., Herault F., Guémené D., Faure J.M., Guy G., 2002c. Effet de la taille du groupe et de la surface allouée pendant la phase de gavage chez le canard mulard. III. Corticostéronémie. 5èmes Journ. Rech. Palm. Foie Gras, 84-87.

Rodenburg T.B., Bracke M.B.M., Berk J., Cooper J., Faure J.M., Guémené D., Guy G., Harlander A., Jones T., Knierim U., Kuhnt K., Pingel H., Reiter K., Servière J., Ruis M.A.W. 2005. Welfare of ducks in European duck husbandry systems. Wld's Poult. Sci. J., 61, 633649.

\section{Résumé}

Différentes espèces et génotypes d'oie et de canards domestiques sont élevés en France à des fins de production de foie gras et/ou de viande. La production d'oie est marginale et les espèces les plus concernées sont respectivement le canard de barbarie (canard à rôtir) et le canard mulard mâle (production de foie gras et de viande). Les conditions d'élevage des palmipèdes ont beaucoup évolué et une grande diversité de systèmes de production coexiste. Les soucis de «bien-être» associés à l'élevage des canards sont, pour l'essentiel, liés aux conditions d'élevage (claustration, cage, densité, sol, accès à l'eau) et au recours à l'épointage et au dégriffage pour prévenir les blessures et les conséquences du picage. La production de foie gras focalise toutefois la majorité des critiques. Pourtant, les données de la littérature et les résultats des travaux conduits, avec des approches expérimentales variées, ne valident pas les arguments permettant d'affirmer que cette pratique nuit gravement au bien-être des palmipèdes, au sens où elle serait source de pathologies, lésions, stress et de douleur. L'élevage en cage individuelle empêche les canards d'adopter certaines postures et d'exprimer certains comportements considérés comme des priorités comportementales, mais l'hébergement collectif idéal reste à mettre au point. Dans ce but, des investigations complémentaires sont entreprises et devraient contribuer à l'amélioration de l'état de bien-être des palmipèdes.

\section{Abstract}

\section{Waterfowl welfare and rearing conditions for meat and foie gras production}

In France, different species and genotypes of waterfowl are reared for the production of foie gras and/or meat. Goose farming is very limited at present and the species reared are Muscovy ducks for meat and male mule ducks (Muscovy and Pekin duck hybrids) for foie gras and meat. Welfare issues in ducks relate mainly to varied rearing conditions (confinement, cage, density, floor, access to water), and the practice of beak and nail trimming to prevent pecking and cannibalism. At present however, it is foie gras production that raises the most controversy, despite the lack of scientific evidence to validate criticisms that force-feeding is detrimental to birds, causing pathology, stress and pain. Rearing in individual cages prevents ducks expressing certain behaviours considered as behavioural priorities. Conditions for multiple cage rearing are still to be defined. With this aim, a number of research programmes are in progress and should contribute to improving the overall conditions of foie gras production.

GUÉMENÉ D., GUY G., MIRABITO L., SERVIÈRE J., FAURE J.-M., 2007. Bien-être et élevage des palmipèdes. INRA Prod. Anim., 20, 53-58. 
